\title{
La adopción de buenas prácticas administrativas en los sectores público y privado como estrategia de prevención de actos de corrupción
}

\author{
JaVier Doria Arrieta ${ }^{1}$
}

\section{RESUMEN}

La suscripción de acuerdos internacionales por Colombia ha motivado medidas legislativas tendientes a prevenir actos de corrupción en los sectores público y privado. Sin embargo, y según las más recientes mediciones internacionales, la percepción de ocurrencia y exposición a prácticas corruptas no deja de ser alta en el país. Ante este panorama, el presente escrito aboga por la adopción de buenas prácticas administrativas, sustentadas en principios de transparencia, eficiencia y eficacia, en los sectores públicos y privado como estrategia de prevención de actos de corrupción.

1 Profesor de postgrado en la catedra de Contratación Estatal, Universidad del Norte, Barranquilla, Colombia, y de la Universidad Tecnológica de Bolívar, de la Escuela Superior de Administración Pública y de la Universidad del Sinú. Asesor, litigante y legal project managment. Abogado, Universidad de Cartagena, Cartagena, Colombia. Magister en Derecho Público, Universidad del Norte, Barranquilla, Colombia. Doctorando en Derecho, Universidad del Norte, Barranquilla, Colombia. Correo-e: javdoria@hotmail.com. Enlace ORCID: https://orcid.org/0000-001-7360-9923. Fecha de recepción: 10 de agosto de 2018. Fecha de modificación: $1 .^{\circ}$ de septiembre de 2018. Fecha de aceptación: 24 de septiembre de 2018. Para citar el artículo: Doria ArRIETA, JAVIER, "La adopción de buenas prácticas administrativas en los sectores público y privado como estrategia de prevención de actos de corrupción", Revista digital de Derecho Administrativo, Universidad Externado de Colombia, n. ${ }^{\circ} 21,2019$, pp. 429-450. DOI: https://doi.org/10.18601/21452946.n21.17 
Palabras clave: corrupción, buenas prácticas, Administración pública, transparencia, eficacia, eficiencia.

\title{
The Implementation of Good Administrative Practices in the Public and Private Sectors as a Strategy for Preventing Acts of Corruption
}

\author{
ABSTRACT
}

International treaties ratified by Colombia have encouraged national legislative measures to prevent acts of corruption in the public and private sectors. However, according to recent international rankings and studies, the perception of risk and incidents of corruption is still high for the country. This paper argues in favor of the implementation of good administrative practices, derived from the principles of transparency, efficacy and efficiency, in the public and private sectors, as a strategy for preventing acts of corruption.

Keywords: Corruption, Good Practices, Public Administration, Transparency, Efficiency, Efficacy.

\section{INTRODUCCIÓN}

El siglo XXI ha marcado la pauta en relación con la necesidad de garantizar el control en la Administración pública con el objetivo de alcanzar la transparencia en las actuaciones del Estado. En tal sentido, el derecho administrativo se muestra como la disciplina responsable de combatir la inmunidad y fortalecer el poder público nacional, bajo el principio de discrecionalidad técnica. De esta manera, este representa y desarrolla un principio de control del poder público inmune, el cual consiste en la existencia de instituciones contraloras, de vigilancia y transparencia del ejercicio del poder público ${ }^{2}$ que se ha ido adaptando, en el caso colombiano, al modelo de Estado vigente con el fin de lograr mecanismos de gobernabilidad y legitimidad a la Administración ${ }^{3}$. Por ende, se observa cómo se plantea la necesidad de una buena administración en el contexto público, enlazada con la buena administración privada, para hacer posible la efectividad y eficacia de la acción de gobernar ${ }^{4}$. El actuar transparente del Estado ha dejado de ser un ejercicio discrecional para convertirse en un paradigma novedoso de administración en la presente

2 José Villar y Francisco Moreno, Congreso Internacional de Derecho Administrativo, Caracas: Universidad Andrés Bello, 2006, p. 374.

3 ManUel ReSTREPO MEDINA, El derecho administrativo en los albores del siglo XXI, Bogotá: Universidad del Rosario, 2007, pp. 12-13. 
centuria ${ }^{5}$, de manera que el buen gobierno sea una práctica del ejecutivo. Esto, en respuesta a la necesidad de cumplimiento de funciones acordes con las normas, reglamentos y políticas que conduzcan a una gestión más honesta, menos corrupta o negligente.

Desde esta óptica, la buena administración, definida como aquella que establece un control efectivo, flexibles a los cambios que acontecen en el ambiente, que regule y facilite la óptima actuación de los diversos elementos que forman parte de la organización ${ }^{6}$, tanto en la práctica privada, como en la pública, tiene virtualidad jurídica. En efecto, ella pretende la aplicación del derecho en las relaciones jurídicas, que conduzca a la observancia de los principios y normativas, inherentes al deber ser de la buena administración, como son los principios de interdicción de la arbitrariedad, economía y eficiencia, objetividad, coordinación y eficacia, que inciden en el desarrollo de la función administrativa. Es allí donde radica la pertinencia del presente análisis, sustentado en la revisión de la literatura científica que aborda el tema.

De esta manera, se examinaron referentes doctrinarios y jurídicos que facilitaron el análisis de información, para cumplir con el objetivo de analizar la adopción de buenas prácticas administrativas en los sectores público y privado como estrategia de prevención de actos de corrupción, en este caso, en Colombia, partiendo de las exigencias derivadas de la buena administración, así como verificar si los mecanismos de buena administración privada puede trasladarse al sector público para disminuir la corrupción al interior del mismo. Para ello, se definen los aspectos referidos a las buenas prácticas administrativas en el sector público; luego, se procede a desglosar la buena administración en el sector privado, para ubicar puntos de encuentro y contribuciones entre ambas y generar un cuerpo de conclusiones acordes con el fin que guía el presente.

\section{BUENAS PRÁCTICAS ADMINISTRATIVAS EN EL SECTOR PÚBLICO}

Para analizar las buenas prácticas administrativas en los sectores público y privado, y establecer la preocupación que ha existido por un tema de especial relevancia en el contexto colombiano actual, se ha considerado que desde las últimas dos décadas del siglo XX se iniciaron trabajos sobre la búsqueda de un derecho a "la buena administración", la cual debía emerger desde el Estado, como garante de servicios mucho más eficientes y efectivos, coordinados y objetivos, tal como se ha vislumbrado en el ordenamiento jurídico interno de cada nación y, para el caso de Colombia, se perfila en la Ley 190 de 1995,

5 Jaime Rodrícuez Arana, Contrataciones públicas en el marco de los derechos sociales fundamentales, Madrid: Instituto Nacional de Administración Pública, 2017, pp. 13-27.

6 Juan Sulbarán G., Aspectos bumanos del control, Mérida: Facultad de los Andes, 1989, pp. $171-172$. 
donde se establecen las normas dirigidas a la preservación de la moralidad en la Administración pública, con la finalidad de erradicar la corrupción administrativa ${ }^{7}$.

Por tanto, la buena Administración pública, entendida como aquella que da fiel cumplimiento a las funciones que le son propias en democracia, centrada en el servicio a la ciudadanía, desarrolladora de un trabajo racional, con plena justificación de sus actuaciones y con respuesta al interés genera $1^{8}$ y la buena administración privada, donde se emplean técnicas y métodos de la ciencia administrativa, cuya interacción conduce a que la pública actúe tutelarmente sobre la segunda; por tanto, de su vinculación surgirían estrategias para prevenir la corrupción, mediante la eficiencia en las operaciones de los actores, conformados por gerentes, supervisores, funcionarios y empleados en general ${ }^{9}$, como práctica vinculada con el daño premeditado, la impostura, la mentira o la falsificación, que lesionan los intereses y valores colectivos, y contribuir con el fortalecimiento y la construcción de una institucionalidad sólida; necesaria para que exista una paz duradera y estable en el territorio nacional ${ }^{10}$.

La buena administración involucra, también, la posibilidad de participar, atendiendo a que este es un derecho a observarse en cada actuación administrativa, partiendo del conjunto de decisiones tomadas por el Estado, a obtener información, a interponer recursos y controvertir las decisiones de las autoridades públicas, a exigir su intervención para solicitar a funcionarios una asistencia oportuna, a fin de prevenir prácticas inadecuadas o corruptas, bajo el entendido que consisten en la malversación de fondos públicos para beneficio personal o ajeno; así como aquellas que implican delitos conexos como tráfico de influencias, uso de información privilegiada, cometidas bajo el amparo de nexos de poder; además, de involucrar a la indebida apropiación de recursos privados en las empresas privadas, por medio de ingentes acciones de ingeniería financiera en organizaciones donde los controles resultan insuficientes e ineficaces ${ }^{11}$; por cuanto, es preciso, la efectiva y pronta participación de los órganos de control del Estado en relación con la prevención de anomalías y hacer posible el imperio de la ley.

A partir de la regulación de la Administración, mediante la definición de controles, se puede garantizar la buena administración como un principio y

7 Congreso de Colombia, Ley 190 de 1995, Diario Oficial, n. ${ }^{\circ}$ 41.878, pp. 1-3.

8 Jaime Rodríguez Arana, El derecho a una buena administración para los ciudadanos, La Coruña: Netbiblo, 2013, p. 245.

9 Manuel Restrepo Medina y Carlos López Cárdena, Adecuación de la Administración pública colombiana al modelo de Estado regulatorio, Bogotá: Universidad del Rosario, 2016, pp. 5-6.

10 Juan Carlos Henao y Aníbal Rafael Zárate, "Estudio preliminar", Corrupción en Colombia t. IV, Corrupción, Estado e instrumentos jurídicos, Bogotá: Universidad Externado de Colombia, 2018, p. 52.

11 Adela Cortina, Corrupción y ética, Bilbao: Universidad de Deusto, 1996, p. 11. 
un derecho de los países ${ }^{12}$. Todo lo cual debe incidir de manera favorable en alcanzar la eficiencia y eficacia de la Administración ${ }^{13}$. Es decir, permitiría alcanzar la eficiencia cuando con los mismos recursos se logra la mayor cantidad de objetivos; mientras que afectaría la eficacia al definir el nivel de prosecución de tales objetivos, todo direccionado para cristalizar la visión de la Administración.

Así se entiende acerca de la pertinencia de una buena administración, para involucrar un conjunto de derechos, garantías y de deberes que se encuentran en los propios estatutos de las instituciones modernas, sustentados en el principio de legalidad y del debido proceso, para hacer posible que haya una buena administración, que responda al interés general, en torno a los derechos humanos, la participación activa que debe tener la ciudadanía, la equidad, la inclusión y los valores y principios de un Estado de derecho líder y protector. Con esto, se aspira a evitar actos de corrupción que afecten la confianza ciudadana en el Estado y en sus servidores públicos. De ahí que sea crucial encauzar el logro de las metas del Estado para reformar y transformar la gestión en el sector público ${ }^{14}$.

En tal sentido, se plantea la necesidad de recuperación de una ética, orientada al servicio a la ciudadanía, propia de la Administración pública ${ }^{15}$. Esto es, la posibilidad de direccionar la actividad de la Administración pública en respuesta a las demandas sociales y económicas que conduzcan a que el Estado se posicione bajo los principios de eficiencia, eficacia y servicio objetivo e imparcial sin influencia de sesgo, prejuicios o tratos diferenciados. De esta forma, la buena administración pública se concibe como un derecho fundamental de la ciudadanía (principios de igualdad, eficacia, celeridad, imparcialidad y moralidad $)^{16}$; al mismo tiempo, se debe convertir en un principio de actuación administrativa ${ }_{i}$ dado que los ciudadanos tienen el derecho a exigir ciertos parámetros en el funcionamiento de la administración, ella, a su vez, está obligada, a diferenciarse en su desempeño diario, por su servicio objetivo al interés general, como se constata en cualquier sistema democrático ${ }^{17}$.

12 Manuel Restrepo Medina, Derecho administrativo. Reflexiones contemporáneas, Bogotá: Universidad del Rosario, 2017, pp. 5-8.

13 Lehidy ANdrea Rosas Tobito, La buena administración del Estado, derecho fundamental sin desarrollo en el ámbito disciplinario, Bogotá: Universidad Militar Nueva Granada, 2015, p. 2.

14 Eber Betanzos Torres, La reforma en materia de combate a la corrupción, México: Fondo de Cultura Económica, 2018, p. 60.

15 Manuel Arenilla Sáez, Crisis y reforma de la administración Pública, La Coruña: Gesbiblo, 2011, p. 109.

16 Corte Constitucional, Sentencia C-826/13: Creación e Implementación de Sistema de Control de Calidad en las Entidades Estatales, Bogotá, 2003, p. 1.

17 Jaime Rodríguez Arana, "La buena administración como principio y como derecho fundamental en Europa", Revista de Derecho y Ciencias Sociales, n. ${ }^{\circ}$ 6, 2013, Bogotá: Misión Jurídica, p. 25. 
Se visualiza, en el planteamiento precedente, que la buena administración pública representa un principio y una obligación que deben asumir las instituciones públicas en cualquier sistema democrático que pretenda alcanzar el desarrollo de este tipo de sistemas de gobierno; también, debe considerar con radicalidad la existencia de la Administración pública, bajo el servicio objetivo al interés general ${ }_{i}$ de este modo, queda entendido que la buena administración pública contribuye con la óptima administración de los recursos del Estado, sustentada en el principio de confianza legítima y de continuidad puedan dar respuesta al interés general ${ }^{18}$.

Además, las instituciones públicas democráticas son del pueblo, en quien reside la soberanía ${ }^{19}$. Por ese motivo, el responsable de la Administración pública debe conocer y practicar el proceso de rendición de cuentas a la ciudadanía en general y buscar la calidad en el servicio objetivo, es decir, la conciencia de institución de servicio esencial a la comunidad ${ }^{20}$, el cual debe regir toda su actuación. Así surge, en diferentes naciones, la "buena administración" en naciones integrantes de la Comunidad Europea, esta se consagra en el artículo 41 de la Carta de los Derechos Fundamentales de la Unión Europea y en la Constitución italiana de 1947; así también en América, en países como la República Bolivariana de Venezuela, donde su Constitución establece el poder ciudadano y la figura del defensor del Pueblo, para garantizar una práctica transparente en la Administración pública ${ }^{21}$, el derecho fundamental del derecho a la buena administración pública, pues el centro que determina la existencia del Estado es la persona, así mismo, la protección y promoción de la dignidad humana y de los mismos derechos que tiene la población como derechos fundamentales. Por ello, queda definida la buena administración pública como la búsqueda de la legitimidad democrática y la eficacia de la actuación pública para cumplir con los derechos de la ciudadanía, encaminando hacia la satisfacción del interés general, para generalizar los beneficios que trae consigo; por tanto, aboga por el respeto y las garantías de los derechos humanos de los ciudadanos, sin ningún tipo de distinción, con el fin de fortalecer la cohesión social ${ }^{22}$.

También comprende la prevención de la corrupción, mediante la creación de un sistema de gestión pública transparente, entendido como una serie de mecanismos, políticas, metodologías, estrategias, técnicas de tipo administrativo y organizacional dirigido a la adecuada gestión del capital humano, físico,

18 Jaime Rodríguez Arana, El ciudadano y el poder público, Madrid: Editorial Reus, 2012, p. 153

19 Corte Constitucional, Sentencia C-245/96: Soberanía Popular, Alcances Jurídico, Políticos, Bogotá, 1996, p. 1.

20 Jaime Rodrícuez Arana, La dimensión ética de la función pública, Madrid: Instituto Nacional de Administración Pública, 2013, p. 58.

21 Carlos Constela, Teoría y práctica del defensor del Pueblo, Bogotá: Editorial Temis, 2010, pp. 221-294.

22 Manuel Arenilla Sáez, óp. cit., p. 109. 
financiero, material y técnico de las entidades de Administración pública ${ }^{23}$ que obstaculice el desarrollo de actos corruptos, producto del uso indebido del poder para beneficio privado o personal, donde funcionarios del sector público o sus allegados se enriquecen ilícitamente ${ }^{24}$; por tanto, una buena administración pública limita los comportamientos conducentes a la obtención de rentas procedentes del sector privado, de los cuales se apropian algunos funcionarios al aceptar "rentas", por medio de sobornos, que son lucrativas para el funcionario, pero que socialmente afectan la estabilidad democrática de las naciones; por esta razón, la buena administración pública en conjunción con la privada, ayuda a la prevención de actos de corrupción en la suscripción de grandes contrataciones con las empresas privadas, producto de la ineficacia y baja calidad de los servicios que brindan los organismos públicos, donde el cliente oferta dinero para que el funcionario cometa actos que son ilegales ${ }^{25}$. Un ejemplo es ExxonMobil Exploration Colombia Limited, la cual se encuentra en Colombia entre las dieciocho Empresas Activas Anticorrupción, para el año 2018.

Del mismo modo, la buena administración pública debe asumir el compromiso con la búsqueda de la calidad de vida de la población. Es así como se encauzará a promover la libertad solidaria de los ciudadanos. Para esto, se debe focalizar en los problemas centrales de la gente, por cuanto debe responder a la sensibilidad social exigida para satisfacer las demandas colectivas ${ }^{26}$. De este modo, se erige la dimensión ética para alcanzar el equilibrio en el cumplimiento de aquellos derechos que resultan inviolables, de tal suerte que sea posible afirmar que lo ético se hace presente en la buena administración cuando se direcciona bajo el servicio objetivo al interés que ha de caracterizar toda acción administrativa y la impronta directiva de los responsables ${ }^{27}$.

Esta protección que debe garantizar el Estado a las personas queda plasmada en el artículo 2 de la Constitución Política, norma que establece dentro de sus fines que se debe respetar la dignidad, garantía y derecho de todas las personas. Por esta razón, se protege a quienes residen en el territorio nacional, no solo desde el punto de vista personal, moral y creencias, sino también en sus bienes, libertades y derechos, a los que el Estado debe responder para cumplir con los derechos y cometidos sociales. Siguiendo con este enfoque, la Administración pública tiene la obligación de aminorar las restricciones en el

23 Congreso de Colombia, Ley 489 de 1998, Bogotá: Diario Oficial, n. ${ }^{\circ} 43.464$ del 30 de diciembre, 1998 , p. 6.

24 Marco Machado, Construyendo ciudadanía, Lima: Forum Solidaridad Perú, 2006, p. 5.

25 Fernando Cepeda Ulloa, Corrupción: Gubernamental, política y empresarial, Cali: Cuellar Editores, 2017, pp. 17-45

26 Luis Miguel Martínez, Alta calidad en la gestión pública, México: Instituto de Administración Pública de México, 2002, pp. 14-19.

27 Jaime Rodríguez Arana, "La buena administración como", óp. cit., p. 25. 
ejercicio de los derechos, a la vez que debe estimular la participación ciudadana en los ámbitos social, cultural, económico y político. Los administradores de la cosa pública deben, por consiguiente, transformarse en servidores de quienes requieren sus servicios, para que la actividad que llevan a cabo sea servicial, diligente y cuidadosa ${ }^{28}$. Es posible entonces concluir que en el ordenamiento jurídico colombiano se le da rango constitucional a la buena administración pública, cuando se reivindica la dignidad del ser humano, donde los valores adquieren un sitial de preponderancia para desarrollar acciones racionales y razonables en el servicio de interés general ${ }^{29}$.

La buena administración pública debe, por lo demás, responder a los siguientes principios: racionalidad, objetividad e imparcialidad, transparencia, coordinación y cooperación, eficiencia y eficacia ${ }^{30}$. La racionalidad se corresponde con el deber de motivar los actos administrativos que se dicten y hacer un uso racional de los recursos públicos, por lo que la actuación debe ser lógica y racional. Este principio también impone el deber de considerar el amparo de la legalidad vigente, por tanto, establece como prohibición dictar actos que sean arbitrarios, lo que debe servir para una apropiada actuación administrativa. Así, ella misma debe propiciar la buena administración privada, pues en interacción harán posible un mejor ejercicio democrático y el respeto a los derechos humanos fundamentales de los hombres ${ }^{31}$.

En relación con la objetividad y la imparcialidad, se atiende a que el personal responsable de cumplir con la administración pública tiene la carga de prevenir la actuación arbitraria, así como cualquier tipo de trato preferente en cualquier circunstancia. Así las cosas, en la motivación del acto administrativo debe estar ausente toda consideración personal. De ahí que sea posible indicar que ella produce resultado criterios uniformes en la adopción de las diferentes decisiones, todo en el marco de la gestión equitativa, racional y de justicia ${ }^{32}$.

En cuanto al principio de transparencia, este se vincula con el acceso a la información de interés general, por tanto, ella debe estar al libre acceso por parte de la ciudadanía ${ }^{33}$. Este mismo principio debe ser compartido por la buena administración privada, como medida de prevención y control en la lucha

28 Corte Constitucional, Constitución Política de Colombia 1991, Bogotá: Consejo Superior de la Judicatura, p. 14.

29 Roosvelt Ospina Sepúlveda, Principio de buena fe y responsabilidad en la Administración pública, Cartagena: Universidad de Cartagena, 2010, pp. 193-194.

30 IZASKun LinaZasoro EspinOza, El derecho a una buena Administración pública, Santiago: Universidad de Chile, 2017, pp. 25-27.

31 Juan Jaramillo Antillón, Principios de gerencia y administración de servicios médicos y bospitales, San José de Costa Rica: Universidad de Costa Rica, 1998, pp. 50-52.

32 Jaime RodríGuez Arana, Derecho administrativo y derechos sociales fundamentales, Madrid: Global Law Press - Editorial Derecho Global, 2015, p. 1.

33 Bernabé Palacin, José Martín y otros, Calidad, transparencia y ética pública, Madrid: Instituto Nacional de Administración Pública, 2017, p. 18. 
contra la corrupción, cuando se trata de la interacción entre ambas; de allí que en las contrataciones del Estado con las empresas privadas deba reinar la transparencia en el proceso y deba suministrarse información a la colectividad para que conozca acerca de la honestidad reinante en el proceso para consolidar una administración eficiente y honrada, garante de la sociedad democrática.

En lo que concierne a la coordinación y cooperación como principios de la buena administración pública, con ellos se pretende alcanzar la integración de la diversidad de las partes en el conjunto del sistema, para prevenir contradicciones. Estos principios motivan a los órganos de la Administración pública a socorrerse con la mutua asistencia, pero siempre bajo la condición de respeto por el ejercicio autónomo de las competencias que cada una de las instituciones administrativas posee ${ }^{34}$.

Otro de los principios mencionados es el de eficiencia, el cual queda vinculado con la conducta eficiente y económica de la Administración pública, esta constituye uno de los parámetros donde se vincula el monto de los recursos empleados con los resultados de los objetivos planeados. De ello se constata que es el deber de actuar de forma racional en el empleo de los recursos públicos ${ }^{35}$.

Abordar el tema de la buena administración pública es una tarea asociada con un conjunto de valores cívicos propios de las características que debe reunir toda sociedad democrática, estos son exigibles al responsable del ejercicio del poder en la Administración pública, partiendo de la concepción de servicio de interés general ${ }_{i}$ por tanto, dicho poder debe reunir como cualidades indispensables que sea compatible, dinámico, atento a la opinión pública, cooperativo, con sentido social, eficaz, eficiente, equilibrado y que responda a la realidad del momento de forma equilibrada, moderada y pluralista ${ }^{36}$. Se interpreta de la precedente idea que la buena administración pública es un factor clave para garantizar la democracia, para ejercer el poder de manera plural, social humana y de manera equilibrada, bajo el principio de racionalidad, desde donde se debe proyectar las distintas maneras de gobernar y administrar.

Además de los planteamientos constitucionales ya nombrados, es posible considerar la Ley 190 de 1995, norma que tiene como objetivo la preservación de la moralidad en la Administración pública, además de fijar una serie de disposiciones para erradicar la corrupción administrativa. En tal sentido, precisa el desarrollo de un sistema de quejas y reclamos en todas las entidades públicas,

34 Pedro Nevado Moreno, Legalidad y buena administración, Bogotá: Universidad Javeriana, 2009, pp. 38-39.

35 Les Metcalfe y Sue Richards, La modernización de la gestión pública, Madrid: Instituto Nacional de Administración Pública, 1989, p. 243.

36 JaIme RODRíGueZ ARANA, "El derecho fundamental a la buena administración y centralidad del ciudadano en el derecho administrativo". Disponible en línea http://derecho.posgrado. unam.mx/congresos/ivci_vmda/ponencias/JaimeRodriguezArana.pdf [consultado el 19 de julio de 2018]. 
según artículo 53, cuya finalidad es favorecer la participación ciudadana en la identificación de las sugerencias que puedan considerarse para una mejor administración pública ${ }^{37}$. De igual modo, concibió, para aquel entonces, la conformación de una Comisión Ciudadana de Lucha contra la Corrupción ${ }^{38}$, con la responsabilidad de brindar asesoramiento al gobierno en su proceso de lucha contra la corrupción, recepción de quejas de los ciudadanos; se convirtió en la instancia desde donde se determinará la vigilancia. Al mismo tiempo, define la pertinencia del desarrollo de investigaciones, partiendo de la consulta de la opinión ciudadana sobre funciones y servicios ofrecidos por las entidades públicas, y con los hallazgos poder definir las medidas de control pertinentes. También establece que la ciudadanía deberá definir los proyectos y planes de acción que se deben considerar para fomentar la participación ciudadana en el ejercicio de control por medio de los mecanismos que defina la Constitución Política y las demás leyes vigentes en Colombia ${ }^{39}$.

Es útil mencionar, para complementar este planteamiento, la Ley 970 de 2005, la cual aprueba la Convención de las Naciones Unidas contra la Corrupción ${ }^{40}$. Esta se aprueba atendiendo a la problemática generalizada en territorio colombiano en relación con la corrupción, la cual causa desequilibrios en la estabilidad y seguridad en la sociedad, ya que desequilibra las instituciones públicas, así como los propios valores democráticos, la ética y la justicia.

De igual modo, la referida reglamentación surge en respuesta a la preocupación generalizada por los nexos entre la corrupción y otras formas de delincuencia organizada, blanqueo de dinero, que desestabilizan la política y el propio progreso naciona $\left.\right|^{41}$; entonces, se pretende el combate contra la corrupción para fortalecer a las instituciones públicas, a través de una buena administración, basada en la eficiencia, eficacia y transparencia en la Administración. Se atiende, de este modo, a una buena administración pública como una vía para brindar a la colectividad un mejor servicio de interés general.

También se encuentra el Decreto 1681 de 1997, donde se define la pertinencia de la lucha contra la corrupción, así como la fusión de la Comisión Nacional Ciudadana para la Lucha contra la Corrupción con la Comisión Nacional para la Moralización, donde se cuenta, entre otras funciones, con

37 Congreso de Colombia, Ley 190 de 1995, óp. cit., p. 14.

38 Comisión Nacional Ciudadana para la Lucha Contra la Corrupción (CNCLC), instancia creada por la Ley 1474 de 2011 con el propósito fundamental de contribuir desde la sociedad civil colombiana a hacer seguimiento a las políticas, programas y acciones formuladas e implementadas por el Estado y el Gobierno Nacional para la prevención, control y sanción de la corrupción.

39 Congreso de Colombia, Ley 190 de 1995, óp. cit., pp. 1-3.

40 Congreso de Colombia, Ley 970 de 2005, Bogotá: Diario Oficial, n. ${ }^{\circ} 45.970$, julio 15 de 2005.

41 José Luis Arriaga Ornelas, "La delincuencia organizada: Análisis de su repercusión en el ejercicio del poder", Revista Criminalidad, vol. 55, n. ${ }^{\circ} 2,2009$, pp. 81-101. 
la vigilancia de la gestión pública, para hacerle frente a situaciones donde la moralidad de la Administración pública pueda ser lesionada, así como para adoptar una estrategia de atención y lucha a favor de la buena administración pública, por medio de acciones tendientes a garantizar la moralidad y prevención de la corrupción ${ }^{42}$.

En síntesis, la buena administración pública se puede concebir como aquel tipo de administración, cuya principal función debe conducir con las funciones que le son inherentes a los sistemas democráticos en relación con el servicio objetivo a la ciudadanía, tomando en cuenta la racionalidad, haciendo justificaciones en torno a las actuaciones que realiza, atendiendo al interés general, el cual reside en la mejora constante e integral de las condiciones de vida de las personas.

En el caso colombiano, la Administración pública se ha visto impactada en forma desfavorable por diferentes eventos, como el caso de la denominada parapolítica $^{43}$, vinculada con los nexos que se establecieron entre carteles y políticos desesperados en la búsqueda de votos y financiamiento, lo que trajo como consecuencia una profunda crisis por la vulneración que impactó la legitimidad de las instituciones públicas y, por ello, de la propia democracia colombiana. De ello, se considera que la buena administración debe conducir a una cada vez más fortalecida democracia, donde reine la transparencia del sistema político, y se implemente una eficaz lucha en contra de la corrupción ${ }^{44}$.

La Administración pública, para que realmente pueda cumplir con su cometido, debe enlazarse con la administración privada, entre ellas debe existir un punto de conexión, por cuanto, muchas veces ellas aspiran alcanzar el mismo propósito que es prevenir la corrupción y ofrecer servicios que satisfagan el interés general de la colectividad.

\section{LA BUENA ADMINISTRACIÓN EN EL SECTOR PRIVADO}

Hoy en día se considera que Colombia ha estado plagada de un sistema de administración que se ha dejado permear por la corrupción, el cual atenta contra la estabilidad económica, la credibilidad en las instituciones, tanto aquellas de carácter público como las privadas, como es el caso que ocupan en este aparte, situación que afecta la estabilidad de la paz y su permanencia en el escenario

42 Presidencia de la República de Colombia, Decreto 1681 de 1997, Bogotá: Diario Oficial, n. ${ }^{\circ} 43.972,1997$, p. 1.

43 Otros ejemplos son los casos de la llamada "Yidis Política", referida a la compra de votos en el Congreso para favorecer la aprobación de la reelección de Álvaro Uribe.

44 Fernando Cepeda Ulloa, Narcotráfico, financiación política y corrupción, Bogotá: Ecoediciones, 2011, p. XXI. 
nacional ${ }^{45}$. Por tanto, se atiende a la necesidad de limitar los espacios a la corrupción, la violencia y el resentimiento que estos puede acarrear. En este contexto, al sector privado se le asigna un rol importante y de trascendencia nacional, pues, a la par de la Administración pública, debe poner en práctica acciones para garantizar la buena administración en el sector privado, dirigidos a la implementación de mecanismos de prevención, detección y denuncia de los posibles actos de corrupción, como acción para combatir este problema ${ }^{46}$.

Esta necesidad se corresponde con lo establecido en la Ley Antisoborno ${ }^{47}$, la cual le otorga facultades a la Superintendencia de Sociedades para sancionar a las personas jurídicas, que por acción u omisión han realizado o propiciado ofrecimientos o entregas a dádivas a funcionarios nacionales o extranjeros. En esta dirección, la intención de la norma es la de promover y regular la construcción de un sistema de cumplimiento anticorrupción por parte de las organizaciones empresariales propias y extranjeras, localizadas en el territorio colombiano, que cumplan funciones de tipo comercial. Por tanto, el fin que se persigue con este tipo de acción es fortalecer las relaciones de confianza entre las organizaciones privadas y el Estado, y fomentar una cultura de legalidad e integridad empresarial, para que ello sea un aporte significativo en beneficio de la competitividad y la productividad privada ${ }^{48}$.

Asimismo, existe en el caso colombiano un marco legal interno para garantizar la buena administración privada, por el cual se debe regir toda práctica que pretenda luchar contra la corrupción, y promover la transparencia al proceso. Es por esto que tales normas atienden al cumplimiento de las principales responsabilidades y a las conductas que se deben prevenir de los particulares, personas naturales, así como de las empresas, para esclarecer el cúmulo de consecuencias de orden jurídico cuando ellas son infringidas. En tal sentido, cabe mencionar la siguiente normativa:

Se tiene, en primer lugar, la Ley de Transparencia y Acceso a la Información Pública (Ley 1712), donde se establecen los estándares de tipo internacional en relación con el derecho que presenta la ciudadanía de contar con acceso a la información, responsabilidad que deben cumplir las organizaciones públicas, así como las organizaciones de carácter privado que ejerzan funciones públicas

45 Andrea Zuleta, La corrupción, su bistoria y sus consecuencias en Colombia, Bogotá: Universidad Militar Nueva Granada, 2015, pp. 5-7.

46 Secretaría de Transparencia, Presidencia de la República, ¿Cómo y por qué implementar u programa empresarial de cumplimiento anticorrupción?, Bogotá: Imprenta Nacional de Colombia, 2016, p. 3.

47 Congreso de Colombia, Ley 1778 de 2016, por la cual se dictan normas sobre la responsabilidad de las personas jurídicas por actos de corrupción transnacional y se dictan otras disposiciones en materia de lucha contra la corrupción, Bogotá: Presidencia de la República de Colombia, 2016, pp. 1-5.

Ibíd. 
en relación con la información vinculada con su actuación, así deberán brindar información como una obligación propia de su desempeño ${ }^{49}$.

De igual modo, se encuentra la Ley 1474 de 2011, Estatuto Anticorrupción, el cual establece las normas que deben seguir las organizaciones empresariales en cumplimiento de una buena administración privada que viabilice la posibilidad de prevención en materia de corrupción. Allí se tipifica el delito de "soborno internacional", las medidas que deben aplicarse a las empresas o personas jurídicas por conductas reñidas con la transparencia que conducen a la corrupción, especialmente empleadas con los administradores o representantes legales que hayan sido beneficiados de delitos contra la Administración pública o que afecten al patrimonio público; estas medidas sancionatorias comprenden multas y penas privativas de la libertad. También, esta normativa establece, en el sector privado, la inclusión de los interventores como sujetos disciplinares, la exclusión de beneficios para aquellos que cometan delitos que vayan en contra de la Administración pública, la responsabilidad para contratistas de construir encargos fiduciarios para todo aquello referente a los anticipos y de invertir exclusivamente tales recursos en el desarrollo del contrato asignado, así mismo, establece la prohibición de contratación con aquellas organizaciones que hayan financiado campañas electorales, la administración desleal y la corrupción privada, entre otros aspectos ${ }^{50}$.

En la misma línea se hace referencia al Conpes 167 de 2013 (Estrategia Nacional de la Política Pública Integral Anticorrupción), donde se logra precisar, en materia de administración privada, la necesidad de incorporar mecanismos para estimular la corresponsabilidad en la prevención de la corrupción, a través de la definición de medidas que guíen los acuerdos de regulación gremial, así como el desarrollo de prácticas de transparencia y la regulación de la responsabilidad de las personas jurídicas para que cumplan fielmente con los estándares que se han fijado en materia de transparencia de la Administración. Lo anterior, por medio de la implementación de registros y sistemas de publicidad, el desarrollo de materiales guía en torno a las buenas prácticas y el reconocimiento de las experiencias de transparencia y lucha en contra de la corrupción $n^{51}$.

También se encuentra el Conpes 3793 de 2013 (Política Nacional Antilavado de Activos y Contra la Financiación del Terrorismo), donde se establece un marco de referencia dirigido a la conformación de las condiciones adecuadas para el sector privado, el cual debe integrarse en la creación de una cultura de

49 Congreso de Colombia, Ley 1712 de 2014, Ley de Transparencia y Acceso a la Información Pública, Bogotá: Presidencia de la República de Colombia, 2014, pp. 1-29.

50 Congreso de Colombia, Ley 1474 de 2011, Estatuto Anticorrupción, Bogotá: Presidencia de la República, 2011, pp. 1-54.

51 Consejo Nacional de Política Económica y Social, Estrategia Nacional de la Política Pública Integral Anticorrupción 167, Bogotá: Presidencia de la República, 2013, pp. 9-18. 
prevención de delitos, con el diseño de estrategias de administración de riesgos en el interior de las organizaciones, a fin de reportar y denunciar cualquier tipo de operación sospechosa ${ }^{52}$.

En el escenario colombiano, es preciso señalar que la buena administración privada ha estado regida por los pactos de transparencia e integridad, definidas por la Secretaría de Transparencia de la Presidencia de la República, los cuales contemplan estrategias que son de especial pertinencia para garantizar un modelo de integridad del empresariado privado, que conforman un mismo sector de negocio, para que adopten un conjunto de principios éticos a ser considerados para efectos de la prevención y disminución de casos de corrupción. Estos pactos son de voluntaria adhesión y la declaración se suscribe entre las organizaciones empresariales firmantes, tomando como testigo a la Secretaría de Transparencia, quien realiza todo el acompañamiento para fortalecer el cumplimiento del compromiso entre las partes, asumir la integridad y transparencia como los ejes transversales que deben regir las relaciones para evitar riesgos de corrupción entre los firmantes. Así mismo, se establece un canal para la consulta, denuncia e información constante acerca de eventos de corrupción, en los que se definen las responsabilidades que se tienen en relación con reportar casos de corrupción, como también la elaboración de planes relacionados con este campo y la conformación de un comité de ética para hacer seguimiento al desarrollo de los compromisos que las partes han asumido en torno a la integridad ${ }^{53}$.

En consideración a lo referido, existe un conjunto de pasos que se inicia con la asignación de un facilitador, quien debe asumir una posición neutral en relación con los intereses de cada una de las organizaciones empresariales involucradas, para contribuir con la estructuración de una iniciativa de corte colectivo, donde se alcancen propósitos que son comunes a los involucrados. Además, se plantea la investigación en torno a los motivos por los cuales cada una de las empresas se suscribe al convenio; luego, se procede a identificar los riesgos presentes en los actos de corrupción común al sector de negocios y/o empresas firmantes. De esta manera, el paso que se menciona a continuación implica precisar las medidas de control para contrarrestar los riesgos previamente identificados; se establecen los compromisos para garantizar la operatividad del pacto, conformando un grupo responsable de la promoción de la iniciativa, así como una secretaría ${ }_{i}$ finalmente, el pacto se somete a ser

52 Consejo Nacional de Política económica Y Social, Política Nacional Antilavado de Activos y Contra la Financiación del Terrorismo 3793, Bogotá: Presidencia de la República, 2013, pp. 8-17.

53 Secretaría de Transparencia, Presidencia de la República, Pactos de Transparencia e Integridad, Sector Privado, 2018. Disponible en línea http://www.secretariatransparencia.gov. co/estrategias/Paginas/pactos-transparencia-integridad.aspx [consultado el 18 de julio de 2018]. 
firmado. Se señala que la Secretaría de Transparencia tendrá la posibilidad de realimentar el pacto, en sus versiones preliminares, así como, deberá hacer el acompañamiento respectivo a todas aquellas organizaciones empresariales que así lo necesiten ${ }^{54}$.

Sobre este punto, cabe mencionar el aporte que debe realizar el sector privado en la prevención de los actos de corrupción, lo cual implica la implementación de buenas prácticas de administración en el interior de las empresas, el establecimiento de códigos de éticas corporativos, la definición de sistemas de vigilancia y auditoría dirigidos al control de desviaciones que pudieran presentarse en el contexto de la organización empresarial ${ }^{55}$. Por tanto, la buena administración privada contribuye con la disminución de casos de corrupción en el interior de las mismas, pero también en la contratación con el Estado, dado que la empresa privada se aboca al cumplimiento de las leyes nacionales, para prevenir cualquier práctica que aliente la corrupción. Así, presentarán informes como acción dirigida a la demostración de la sinceridad y seriedad de la administración privada en la lucha contra la corrupción y su compromiso con valores de especial relevancia como lo son la rendición de cuentas, transparencia, e integridad, todo lo cual redunda en reputación y credibilidad en la administración privada, dadas las buenas prácticas anticorrupción.

De igual modo, esto implica el desarrollo de programas de cumplimiento o compliance de las normas y regulaciones a las que da lugar la legislación para contar con una administración transparente, donde se refuerzan mecanismos de control preventivo en materias como contrataciones, y la excelencia gestora antifraude y que al contratar, por ejemplo, con el Estado, puedan en conjunto luchar por un control efectivo, transparente, rápido y oportuno; de esta forma, se podrá descongestionar la Administración pública y privada ${ }^{56}$.

\section{RELACIÓN ENTRE LAS BUENAS PRÁCTICAS ADMINISTRATIVAS EN EL SECTOR PÚBLICO Y ADMINISTRACIÓN EN EL SECTOR PRIVADO}

Una vez establecida la doctrina en cuanto a la buena administración pública y la buena administración privada, se ha considerado pertinente definir cuáles son los puntos de encuentro entre ambas, para relacionarlas en función de garantizar prácticas anticorrupción en el escenario colombiano. Así, las medidas que se adopten en el campo privado podrán surtir efectos positivos en el ámbito público y viceversa.

Ibíd.

55 Oficina de las Naciones Unidas Contra la Droga y el Delito, Programa Anticorrupción de Ética y Cumplimiento para las Empresas, Nueva York: Naciones Unidas, 2013, pp. 19-31.

56 Abraham Castro Moreno y Pilar Otero González, Prevención y tratamiento punitivo de la corrupción en la contratación pública y privada, Madrid: Editorial Dykinson, 2016, pp. 7-15. 
Sin embargo, es pertinente aclarar que la Administración pública es aquella responsable de garantizar el adecuado funcionamiento y administración de los servicios y programas dirigidos a la colectividad para el apropiado manejo de los recursos del Estado, para brindar la mayor cantidad de beneficios a la colectividad para fortalecer la calidad de vida de la gente en el ámbito local, regional y nacional. En lo dicho se constata que es el Estado el órgano rector de la Administración pública, por medio de diferentes organizaciones responsables de brindar atención a la población. En el caso de la administración privada, ella tiene como objetivo propiciar el adecuado funcionamiento y administración de las empresas, las cuales tienen fines de lucro, y que se diseminan a lo largo y ancho del territorio de una nación. Estas se financian de capital privado. En tal sentido, están dirigidas a la gestión interna de las organizaciones; ellas deben guiarse por normas y leyes impuestas por el Estado en relación con la administración de sueldos y salarios, contrataciones, beneficios laborales, seguridad social, medio ambiente de trabajo, entre otros ${ }^{57}$.

A pesar que ambas tienen fines diferentes, no es menos cierto que ambas tienen relación en lo que concierne a la buena administración que deben llevar cuando interactúan, dado que la buena administración pública se desempeña tutelarmente sobre la privada, debido a que su radio de actuación involucra a todas las organizaciones que hacen vida social.

Tomando en consideración lo dicho, existe una total vinculación de ambos tipos de administración, pues como lo refiere a la eficiencia eficacia que ambas deben reunir para el logro de los objetivos que procuran alcanzar. Por tanto, ellas deben basarse en las buenas prácticas, cumpliendo las normas que la Administración pública ha establecido para la privada y la privada tomando en cuenta que, al contratar con el Estado, dicho proceso debe basarse en la transparencia, la legalidad para servir bien, para lograr los fines que se plantean ${ }^{58}$.

De esta manera, la buena administración pública y privada, en el caso colombiano, es un derecho de los ciudadanos, para satisfacer el interés general ${ }_{i}$ la privada es de especial relevancia nacional, por contar con el capital necesario para acatar las leyes, tanto las civiles como las mercantiles, debe cumplir la legislación que establece el Estado. Al vincularla con la buena administración pública se entiende que, al desarrollar contrataciones con las empresas privadas, el Estado deberá cumplir con procesos licitatorios, realizado públicamente con la finalidad de convocar a todas aquellas organizaciones interesadas en presentar ofertas y, bajo un proceso transparente seleccionar aquella que resulte más favorable ${ }^{59}$.

Aleskey Herrera Robles, Aspectos generales del derecho administrativo colombiano, Barranquilla: Universidad del Norte, 2012, p. 601. 
Por tanto, se trata de una relación guiada por la regulación de la actuación de las partes, en torno a criterios de idoneidad moral, tanto financiera como técnica, donde se plantea una evaluación de las empresas que licitan para escoger aquellas que reúnan los requisitos objetivos mínimos requeridos, que brinden las mayores garantías al interés general que debe tutelar la administración pública.

Al mismo tiempo, ellas se vinculan en que ambas requieren centralización y unidad de acción, justicia y equidad en su actuación, por medio de cualquier actuación, implica que deben existir mecanismos de control, para definir medidas de preventivas de actos de corrupción, a través de funciones de inspección, sobre todo en la contratación pública con la privada, mediante una total independencia y transparencia de actuación de las partes ${ }^{60}$.

En las relaciones que definen la buena administración pública y la buena administración privada se establece un vínculo mediado por la necesidad de precisar programas de rendición de cuentas entra las partes, cuando ambas determinan algún tipo de vínculos, donde la empresa privada es contratada con fines de ofrecer servicios públicos. Esto significa abrir oportunidades para que la ciudadanía en general pueda contar con información objetiva, confiable y transparente en torno al trabajo que realizan en conjunto, hecho que se convierte en una estrategia anticorrupción.

De igual modo, la buena administración pública y la privada, en sus relaciones, al responder al interés general, tienen la responsabilidad de conformar índices internos y externos de rendición de cuentas, donde se cumplan estándares internacionales de contratación, entre ambas, con procedimientos regulados, con la presencia de organismos imparciales, sin conexión con agrupaciones de carácter político y sin nexos con funcionarios públicos, que se encarguen de la certificación del proceso, lo que ayuda a controlar y limitar el soborno de las empresas transnacionales con personeros de la administración pública ${ }^{61}$.

En tal sentido, la relación que guía a ambos tipos de administración debe garantizar el cumplimiento de regulaciones donde la interacción de la Administración pública con las grandes corporaciones o empresas privadas, dedicadas a la contratación con el Estado, se apoye en las buenas prácticas, como estrategia central para crear una cultura ética anticorrupción a la cual se vayan adhiriendo todas aquellas organizaciones que desean desarrollar actividades libres de sobornos, tráfico de influencias, extorsiones, fraudes, malversación de fondos, entre otros. 


\section{CONCLUSIONES}

Las buenas prácticas de la administración pública deben estar dirigidas a garantizar una administración transparente en Colombia ${ }_{i}$ por cuanto estas representan una clave fundamental para contar con la formación ética de los funcionarios y dé respuesta al interés general, como factores que deben contribuir con la prevención de actos de corrupción, en el escenario nacional colombiano, elemento clave como estrategia en la lucha anticorruptiva.

Es en este ámbito donde la buena administración pública hace posible el mejoramiento del alcance y funcionalidad, siempre vinculado con el interés general, el cual conduce al cumplimiento del derecho de la ciudadanía, donde el ciudadano no sea un centro receptor de políticas públicas, sino que sean actores participativos en la adecuada administración. Así, se vislumbra el Estado social y democrático de derecho, que contribuya con la conformación de las condiciones apropiadas para determinar la libertad solidaria de las personas en las que se integran removiendo cualquier obstáculo para su efectiva realización.

En este orden de ideas, la buena administración tiene la intención de involucrar en el desarrollo de una conducta ética para quienes laboran dentro de los órganos de Administración pública. Por tanto, es urgente la creación de un código de ética, que sea cumplido por quienes administran la cosa pública, con la finalidad de satisfacer el interés general de la ciudadanía, para evitar disfunción o vicio en lo que se ha tildado como "mala administración"62.

Quienes son responsables de buena administración pública deben asumir un desempeño cónsono con principios de equidad y objetividad, así como en la prestación de servicios eficientes, eficaces y oportunos, en materia de regulación de la Administración pública. Por tanto, la pertinencia dentro de las instituciones públicas el desarrollo de procedimientos que contribuyan con la pulcritud en el uso de sus recursos. Dicha pulcritud implica el cumplimiento de principios de eficiencia y eficacia, para garantizar el logro adecuado de los objetivos planteados por la Administración pública.

De igual manera, en torno a la buena administración pública y privada, el Estado debe asumir su responsabilidad y compromiso en la necesidad de propiciar la conformación de espacios donde la administración enfrente las necesidades ciudadanas para estimular un Estado de bienestar que eleve la calidad de vida. Es por esto que se concibe que la buena administración debe contar con instrumentos legales y normativos, que viabilicen la oportuna prestación de servicios eficientes y eficaces, para disminuir la corrupción, por medio de controles que den respuesta a las necesidades presentes en la prevención de actos corruptos y el mejoramiento de la transparencia.

62 Manuel Villoria Mendieta, Ética pública y buen gobierno, Madrid: Instituto Nacional de Administración Pública, 2015, pp. 18-34. 
En consecuencia, se plantea que los funcionarios deben basarse en los valores fundamentales, como honradez, honestidad, fidelidad, justicia y respeto a la dignidad de la persona, entre otros, en los cuales se sustenta el cuerpo jurídico que regula la buena administración, centrados en la integridad, transparencia y responsabilidad, adoptados por los organismos del Estado, todo ello para hacer un buen gobierno, que en conjunto con la administración privada beneficien su reputación, en cumplimiento de programas anticorrupción.

Cabe referir que se plantea la necesidad de aplicar códigos ética para quienes cumplen funciones en la administración pública y privada. Esto, con el fin de contribuir con las buenas prácticas a llevar al interior de las organizaciones. De este modo, se inducirá a la conformación de una cultura preventiva a todo aquel involucrado en tareas susceptibles de actos de corrupción, tales como funcionarios públicos, ciudadanos, empresarios y políticos, para conformar una cultura de transparencia en todas las actuaciones dirigidas al interés general. Ello, a su vez, debe estar acompañado de una correcta inversión de los recursos del Estado, por medio de contrataciones que reúnan los requisitos de adjudicación, pero que también se sustenten en los principios establecidos como parte de la buena administración.

En atención a lo escrito, la adopción de buenas prácticas de administración pública y privada implica que den respuesta pronta, oportuna, eficiente y eficaz, y estén sustentadas en la ética, la transparencia y el derecho a la información por parte de la ciudadanía, que desea estar enterada de los vínculos que se puedan establecer entre las partes. Por ejemplo, en casos donde se relaciona lo público con lo privado, la acción a seguir será el aseguramiento de la legalidad en la administración, como instrumento de ejercicio de la prevención de actos de corrupción; por eso, se propicia la responsabilidad, eficacia y capacidad de los intereses de las partes involucradas, para propiciar el consenso y encontrar mecanismos de control que doten a ambas administraciones de un código ético que fortalezca el espíritu democrático en Colombia.

\section{BIBLIOGRAFÍA}

Arenilla Sáez, Manuel. Crisis y reforma de la Administración pública, La Coruña: Netbiblo, 2011.

Arriaga Ornelas, José Luis. "La delincuencia organizada: Análisis de su repercusión en el ejercicio del poder, Revista Criminalidad, vol. 55, n. ${ }^{\circ}$ 2, 2009.

Betanzos Torres, Eber. La reforma en materia de combate a la corrupción, México: Fondo de Cultura Económica, 2018.

Castro Moreno, Abraham y Pilar Otero González. Prevención y tratamiento punitivo de la corrupción en la contratación pública y privada, Madrid: Editorial Dykinson, 2016. 
Cepeda Ulloa, Fernando. Corrupción: Gubernamental, política y empresarial, Cali: Cuellar Editores, 2017, pp. 17-45.

Cepeda Ulloa, Fernando. Narcotráfico, financiación política y corrupción, Bogotá: Ecoediciones, 2011.

Consejo Nacional de Política Económica y Social. Estrategia Nacional de la Política Pública Integral Anticorrupción 167, Bogotá: Presidencia de la República, 2013.

Consejo Nacional de Política económica Y Social. Política Nacional Antilavado de Activos y Contra la Financiación del Terrorismo 3793, Bogotá: Presidencia de la República, 2013.

Constela, Carlos. Teoría y práctica del defensor del Pueblo, Bogotá: Editorial Temis, 2010.

Corte Constitucional, Sentencia C-245/96: Soberanía Popular, Alcances Jurídico, Políticos, Bogotá, 1996.

Corte Constitucional, Sentencia C-826/13: Creación e Implementación de Sistema de Control de Calidad en las Entidades Estatales, Bogotá, 2003.

Cortina, Adela. Corrupción y ética, Bilbao: Universidad de Deusto, 1996.

Henao, Juan Carlos y Aníbal Rafael Zárate. "Estudio preliminar", en Corrupción en Colombia, t. IV, Corrupción, Estado e instrumentos jurídicos, Bogotá: Universidad Externado de Colombia, 2018

Herrera Robles, Aleskey. Aspectos generales del derecho administrativo colombiano, Barranquilla: Universidad del Norte, 2012.

Igarza Funes, Gustavo. La buena administración y la negociación colectiva, Montevideo: Universidad de Montevideo, 2016.

Jaramillo Antillón, Juan. Principios de gerencia y administración de servicios médicos y hospitales, San José de Costa Rica: Universidad de Costa Rica, 1998.

Jiménez Arana, Armando. Míster, cpor qué nosotros?, Sevilla: Punto Rojos Libros, 2013.

LinaZasoro Espinoza, IZASKun. El derecho a una buena Administración pública, Santiago: Universidad de Chile, 2017.

Machado, Marco. Construyendo ciudadanía, Lima: Forum Solidaridad Perú, 2006.

Martínez, Luis Miguel. Alta calidad en la gestión pública, México: Instituto de Administración Pública de México, 2002. 
Metcalfe Les y Sue Richards. La modernización de la gestión pública, Madrid: Instituto Nacional de Administración Pública, 1989.

Nevado Moreno, Pedro. Legalidad y buena administración, Bogotá: Universidad Javeriana, 2009.

Oficina de las Naciones Unidas Contra la Droga y el Delito. Programa Anticorrupción de Ética y Cumplimiento para las Empresas, Nueva York: Naciones Unidas, 2013.

Ospina Sepúlveda, Roosvelt. Principio de buena fe y responsabilidad en la Administración pública, Cartagena: Universidad de Cartagena, 2010.

Palacin, Bernabé, José Martín y otros. Calidad, transparencia y ética pública, Madrid: Instituto Nacional de Administración Pública, 2017.

Restrepo Medina, Manuel. Derecho administrativo. Reflexiones contemporáneas, Bogotá: Universidad del Rosario, 2017.

Restrepo Medina, Manuel. El derecho administrativo en los albores del siglo XXI, Bogotá: Universidad del Rosario, 2007.

Restrepo Medina, Manuel y Carlos López Cárdena. Adecuación de la Administración pública colombiana al modelo de Estado regulatorio, Bogotá: Universidad del Rosario, 2016.

Rodríguez Arana, Jaime. Contrataciones públicas en el marco de los derechos sociales fundamentales, Madrid: Instituto Nacional de Administración Pública, 2017.

Rodríguez Arana, Jaime. Derecho administrativo y derechos sociales fundamentales, Madrid: Global Law Press - Editorial Derecho Global, 2015.

Rodríguez Arana, Jaime. El ciudadano y el poder público, Madrid: Editorial Reus, 2012.

Rodríguez Arana, Jaime. El derecho a una buena administración para los ciudadanos, La Coruña: Netbiblo, 2013.

Rodríguez Arana, Jaime. "El derecho fundamental a la buena administración y centralidad del ciudadano en el derecho administrativo". Disponible en línea http:// derecho.posgrado.unam.mx/congresos/ivci_vmda/ponencias/JaimeRodriguezArana.pdf. [Consultado el 19 de julio de 2018].

Rodríguez Arana, Jaime. "La buena administración como principio y como derecho fundamental en Europa", Revista de Derecho y Ciencias Sociales, n. 6, Bogotá: Misión Jurídica, 2013.

Rodríguez Arana, Jaime. La dimensión ética de la función pública, Madrid: Instituto Nacional de Administración Pública, 2013. 
Rosas Tobito, LeHidy Andrea. La buena administración del Estado, derecho fundamental sin desarrollo en el ámbito disciplinario, Bogotá: Universidad Militar Nueva Granada, 2015.

Ruiz-TAgle, Eduardo. Gobernabilidad democrática, Santiago: Editorial Andrés Bello, 1997.

Sandoval, Irma Edendira. Corrupción y transparencia. Debatiendo las fronteras entre Estado, mercado y sociedad, México: Editorial Siglo XXI, 2009.

Secretaría de Transparencia, Presidencia de la República. ¿Cómo y por qué implementar u programa empresarial de cumplimiento anticorrupción?, Bogotá: Imprenta Nacional de Colombia, 2016.

Secretaría de Transparencia, Presidencia de la República. Pactos de Transparencia e Integridad, Sector Privado, 2018. Disponible en línea http://www.secretariatransparencia.gov.co/estrategias/Paginas/pactos-transparencia-integridad.aspx. [Consultado el 18 de julio de 2018].

Sulbarán G., Juan. Aspectos bumanos del control, Mérida: Facultad de los Andes, 1989.

Villar, José y Francisco Moreno. Congreso Internacional de Derecho Administrativo, Caracas: Universidad Andrés Bello, 2006.

Villoria Mendieta, Manuel. Ética pública y buen gobierno, Madrid: Instituto Nacional de Administración Pública, 2015.

Zuleta, Andrea. La corrupción, su bistoria y sus consecuencias en Colombia, Bogotá: Universidad Militar Nueva Granada, 2015. 